\title{
Caller sex and orientation influence spectral characteristics of "two-voice" stereotyped calls produced by free-ranging killer whales
}

\author{
Patrick J. O. Miller ${ }^{\mathrm{a})}$ \\ School of Biology, University of Saint Andrews, St. Andrews, Fife, KY16 9LB, United Kingdom; \\ Laboratory for Computer Science, Massachusetts Institute of Technology, Cambridge, Massachusetts 02139 \\ and Biology Department, Woods Hole Oceanographic Institution, Woods Hole, Massachusetts 02543
}

Filipa I. P. Samarra and Aurélie D. Perthuison

School of Biology, University of Saint Andrews, St. Andrews, Fife, KY16 9LB, United Kingdom

(Received 14 September 2006; revised 7 March 2007; accepted 12 March 2007)

\begin{abstract}
This study investigates how particular received spectral characteristics of stereotyped calls of sexually dimorphic adult killer whales may be influenced by caller sex, orientation, and range. Calls were ascribed to individuals during natural behavior using a towed beamforming array. The fundamental frequency of both high-frequency and low-frequency components did not differ consistently by sex. The ratio of peak energy within the fundamental of the high-frequency component relative to summed peak energy in the first two low-frequency component harmonics, and the number of modulation bands off the high-frequency component, were significantly greater when whales were oriented towards the array, while range and adult sex had little effect. In contrast, the ratio of peak energy in the first versus second harmonics of the low-frequency component was greater in calls produced by adult females than adult males, while orientation and range had little effect. The dispersion of energy across harmonics has been shown to relate to body size or sex in terrestrial species, but pressure effects during diving are thought to make such a signal unreliable in diving animals. The observed spectral differences by signaler sex and orientation suggest that these types of information may be transmitted acoustically by freely diving killer whales. () 2007 Acoustical Society of America. [DOI: 10.1121/1.2722056]
\end{abstract}

PACS number(s): 43.80.Ka [WWA]

Pages: $3932-3937$

\section{INTRODUCTION}

Animal sounds are shaped by the characteristics of both the source and associated resonant structures, and these are constrained by their size and shape (Bradbury and Vehrencamp, 1998). Variation in energy dispersion across signal harmonics has been attributed to body size or vocal tract length (Fitch, 1997), though sex differences may be greater than expected from the direct effect of body size in human speech (Rendall et al., 2005). Voice features such as formant frequency dispersion and sometimes pitch (called $\mathrm{F}_{0}$ in human speech) can vary substantially between calling individuals. For example, dominant frequency and pulse rate (pitch) of calls produced by cricket frogs (Acris crepitans) correlate strongly with body size (McClelland et al., 1996). Relative maximum levels of harmonics and maximum echolocation call frequency differ between individuals and families in the big brown bat (Eptesicus fuscus; Masters et al., 1995).

Fish-eating killer whales (Orcinus orca) produce calls with stereotyped time-frequency contours (Ford, 1991), and many calls contain two independently modulated contours (referred to as "two-voice calls") each with multiple harmonics (Fig. 1; Hoelzel and Osborne, 1986; Miller and Bain, 2000; Miller, 2002). Odontocete cetaceans possess a pair of nasal lips that could be used to generate two independently

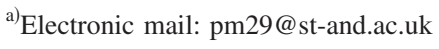

controlled signals (Cranford, 2000). The low-frequency component (LFC) of killer whale calls is thought to be a burstpulse sound with the pitch, or fundamental frequency, generated by the pulse-repetition rate. Gradations in the LFC pulse rate range from slow-rate intervals in which individual pulses are apparent, often observed at the start of calls, to high-rate intervals, which sound more tonal. Though little is known about the high-frequency component (HFC), calls can contain modulation sidebands that appear above and below the HFC (Fig. 1). In all calls that we have inspected with sidebands, they are always spaced from the HFC by precise multiples of the frequency of the LFC (Fig. 1), indicating that the source mechanism driving the LFC also modulates the HFC.

Time-frequency contours of both components of killer whale calls differ primarily by social group (Ford, 1991; Deecke et al., 2000; Miller and Bain, 2000), with only minor differences between individuals within the same matrilineal group (Nousek et al., 2006). Extreme body-size dimorphism (adult males are $\frac{1}{3}$ longer and have twice the volume as adult females; Matkin and Leatherwood, 1986) may provide an anatomical basis for spectral differences in acoustic signals by sex. Because the time-frequency contour is primarily group specific, such differences might be useful as a cue to aid within-group recognition or to identify caller sex.

Unlike terrestrial animals that live in static ambient pressure, breath-hold divers can routinely experience severe pres- 

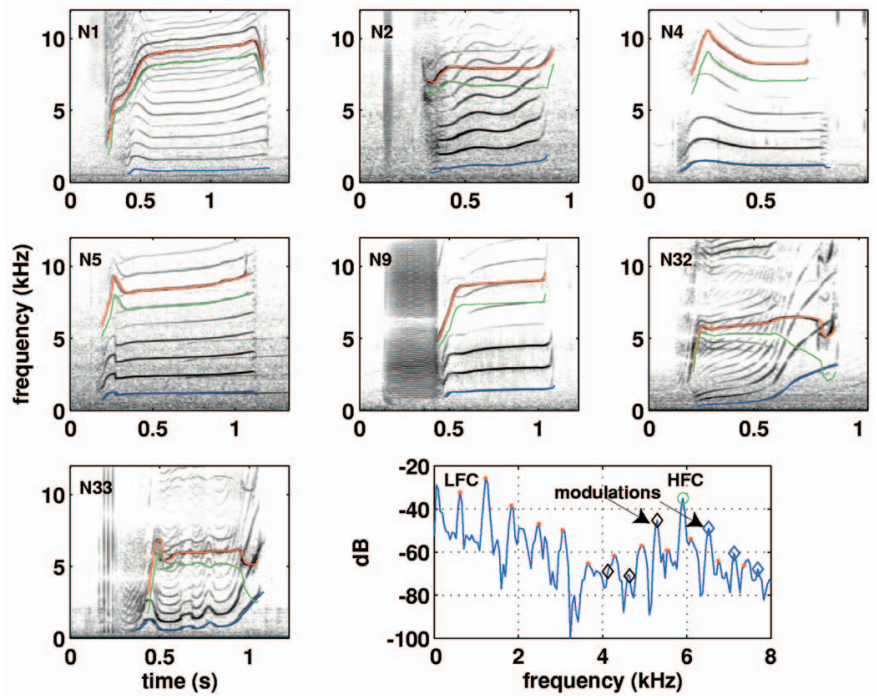

FIG. 1. Spectrograms of the seven call types analyzed. The time-frequency contour of the LFC fundamental is shown in blue, and the HFC fundamental in red. Note the presence of sidebands above and below the HFC. The frequency positions of these bands correspond precisely to the $\mathrm{HFC} \pm$ multiples of the LFC. The HFC contour minus the LFC contour is shown in green and overlaps a modulation band in all call types except N9. Lower right: power spectral density plot of the N33 call at time point 0.6 s. Note peaks corresponding to the LFC (red dot), HFC (green circle), and HFC modulations (black and blue diamonds). We recorded the peak levels of the first two harmonics of the LFC and the fundamental of the HFC.

sure changes. The acoustic properties of air-filled structures should be affected by reduction of total air volume and by increased density of the air when under pressure (Bradbury and Vehrencamp, 1998). This fact has led to the conjecture that anatomical voice cues that rely on passive resonant effects of air cavities may not be reliable in diving animals (Tyack, 1991; Janik and Slater, 2000; Tyack and Miller, 2002). The frequency pattern of response whistles produced by diving beluga whales (Delphinapterus leucas) in a training task was shown to change strongly with depth though the fundamental frequency was unchanged (Ridgway et al., 2001). The pitch of calls produced by diving seals did not vary with caller depth (Moors and Terhune, 2005), suggesting that the fundamental frequency of calls may be more effectively controlled than the dispersion of energy across harmonics. Diving may thereby select for active control of acoustic features used for individual or sex recognition, such as frequency modulation recently shown to be important in bottlenose dolphin signature whistle recognition (Janik et al., 2006). However, it is possible that calling divers could compensate for pressure effects on air resonant cavities (Amundin, 1991) or that anatomical voice cues are generated by tissue-borne resonances that are less affected by depth (Cranford et al., 1996). It is therefore valuable to explore whether acoustic features that might be shaped by resonant effects, such as the distribution of energy across frequencies, differ consistently by the signaler sex or body size of free-ranging breath-hold divers.

We consider here how the relative intensity of harmonics in both low- and high-frequency components and number of HFC modulation sidebands are influenced by adult signaler sex in calls produced by freely diving adult killer whales. Signaler range and orientation relative to the receiver must be considered in analysis of frequency spectra of sounds from free-ranging animals, and signaler range and orientation are also potentially useful to communicate in groupliving mobile animals (Miller, 2002; Lammers and Au, 2003). The key question we address is whether the spectra of calls received from freely diving whales correlate with sex, orientation, and range of the signaler. Such a relationship would demonstrate that the influence of these signaler characteristics on the communication signals of killer whales can exist and be detected during natural diving behavior.

\section{METHODS}

Recordings were made during August and September 1998 and 1999 off Vancouver Island, Canada, using a beamforming array towed from an $11-\mathrm{m}$ research vessel. This system allows calls to be ascribed to a focal animal when it is separated in azimuth from other group members by $20 \mathrm{deg}$ or more (Miller and Tyack, 1998; Miller et al., 2004). Each time the focal animal surfaced to breathe, we recorded its identification based on dorsal fin and saddle patch shapes (Ford et al., 2000) as well as its orientation and range to the recording array. Animals were classified as juvenile (J) if they were estimated to be under 15 years old or as adult male (AM) or adult female (AF) if otherwise (Olesiuk et al., 1990). The 16-bit, 48-kHz sampling rate acoustic recordings were transferred from Tascam recorders to a computer using Cool Edit Pro.

Only stereotyped calls produced by the identified focal whale were used in the analysis. The orientation of the focal animal at the time a call was received was estimated by interpolating the observed orientations during the surfacings before, and after, production of the call. To reduce errors in the orientation estimate during call production, calls were not included if these were produced between two surfacings for which the observed orientations changed by more than 120 deg. Left- and right-side off-axis orientations were combined, and then converted to radians with values ranging from zero for animals oriented directly toward the array to $\pi$ for animals oriented directly away from the array. Stereotyped calls were classified to type using visual analysis of spectrograms and aural recognition (Ford, 1987; Miller et al., 2004). We only considered call types with both high- and low-frequency components, which resulted in the inclusion of seven call types: N1, N2, N4, N5, N9, N32, and N33 (Fig. $1)$.

We used a trace of the LFC and HFC contours, obtained using a pitch tracking algorithm (Wang and Seneff, 2000), to identify peaks corresponding to the LFC harmonics and the HFC peak (Fig. 1). Each call was divided into 0.01-s time intervals for which a power density spectrum was created and the peaks identified automatically. Because the fundamental frequency, or pitch, of killer whale calls is quite high in most portions of calls, formant patterns could not be directly observed as is possible in analysis of human speech. Most of the energy in the low-frequency component (LFC) occurred in the first two harmonics (the first harmonic refers 


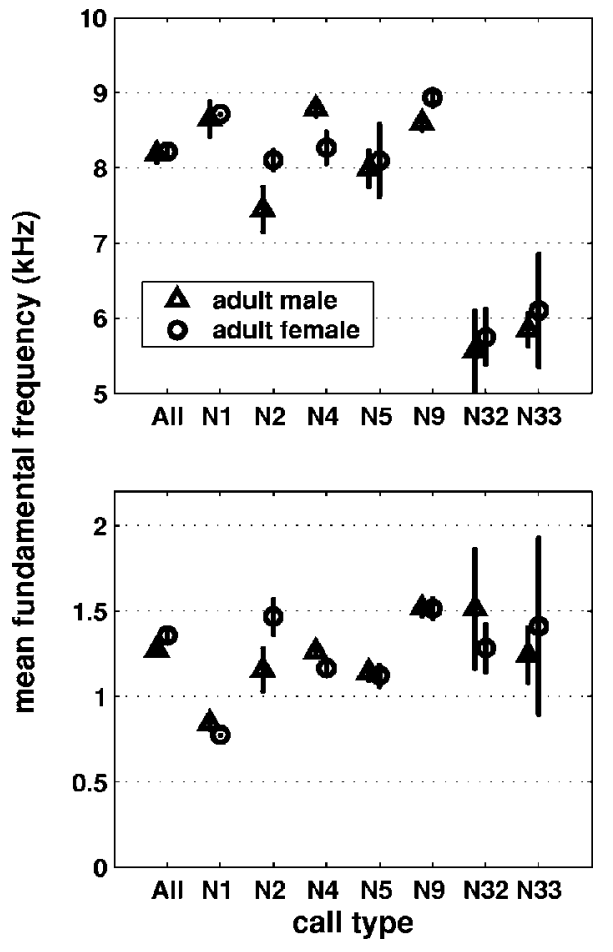

FIG. 2. Mean fundamental frequency of the HFC (top) and LFC (bottom) of calls produced by adult females and adult males. Vertical bars represent \pm 2 standard errors (s.e.) about the mean. Note that there is no consistent pattern by which mean fundamental frequency varies by sex across the different call types.

to the fundamental frequency contour), but harmonics up to the fifth could commonly be measured. Each peak within each interval was inspected by eye to assure that a single, clear peak emerged from the background noise, and that the peak frequency corresponded with the frequency of the contour at that point in time. Peaks within $5 \mathrm{~dB}$ of the noise floor were rejected. Calls were rejected if they did not include at least ten usable intervals. We calculated the average fundamental frequency for LFC and HFC contours, the ratio in intensity between the first and the second LFC harmonics across each call (LFC h2/h1), and the intensity ratio between the HFC and the first two harmonics of the LFC (HFC/LFC). For each call we also counted the maximum number of modulation bands above and below the HFC fundamental that were visible for at least half the call duration.

Statistical analyses were conducted using linear mixed effects models created in $\mathrm{R}$ ( $\mathrm{R}$ Development Core Team, 2004). Linear mixed effects models differ from straightforward linear models in that these are used when the data are grouped according to one or more classification factors, allowing for the incorporation of both effects that influence the mean (fixed factors) and effects that influence the variance (random factors) of the response variable (Pinheiro and Bates, 2000). Fundamental frequency differs by call type (Fig. 2). An analysis of the variation within-calls, using each call number as a random factor, indicated that fundamental frequency of the LFC influences the relative intensity of the first two LFC harmonics $\left(t_{20465}=-11.88, p<0.001\right)$, and that HFC fundamental frequency influences the level of the HFC relative to $\mathrm{LFC}\left(t_{6152}=2.53 ; p=0.011\right)$. This matches the pre- dicted behavior of a resonant system excited with different input frequencies. Therefore, the different fundamental frequency contents within the various call types could be predicted to influence the relative intensities of the LFC harmonics and the LFC relative to the HFC. Different call types also differ in the number of HFC modulation bands (Fig. 1). For this reason, call type is treated as a random factor in the models that relate spectral intensity to orientation, range, and adult sex. The mixed model accounted for a significantly greater amount of the variation in the data than a simple linear model $(P<0.0001)$, justifying the use of this somewhat more complex model design. An autocorrelation term was added to each model as some degree of temporal correlation was present in the data.

Two models were run to test whether LFC and HFC fundamental frequencies differed significantly by sex of the caller. In a second analysis, three models related the intensity ratio of the first two harmonics of the LFC (LFC h2/h1), the ratio of the $\mathrm{HFC}$ to the summed intensity of the first two harmonics of the LFC (HFC/LFC), and the number of HFC modulations to the orientation, range, and age-sex class of the signaler. Range was log transformed. The dependent variables used in the spectral analysis showed low levels of intercorrelation (HFC/LFC with LFC h2/h1: $r=0.126$; HFC/ LFC with no. of HFC sidebands: $r=0.131$; LFC h2/h1 with no. of HFC sidebands: $r=-0.19)$. Such low correlation levels justify the use of separate models for each dependent variable.

In the mixed models used here, the degrees of freedom equal the total sample of calls minus the number of parameters estimated in the model, and again minus the number of groups within the random variable (Pinheiro and Bates, 2000). As five separate models were run, we used the Bonferroni correction to set the level of significance within each model to 0.01 , which assures an experimental probability of Type-I error of 0.05 .

\section{RESULTS}

The process of data selection resulted in a total of 335 calls of seven types produced by 16 individual whales being usable to compare the intensities of the first two harmonics of the LFC (Table I). Due to a very small sample size (17 calls from two juveniles), we removed juveniles from all analyses. This yielded 318 calls total of which 210 were produced by nine adult males and 108 by five adult females. Of these 318 calls, 194 had sufficient peaks to be included in analysis of the HFC, the others being rejected either because the fundamental of the HFC was within $5 \mathrm{~dB}$ of the noise floor or overlapped a harmonic of the LFC. Of these 194, 125 were produced by the nine adult males and 69 were produced by the adult females.

Sex of the caller did not consistently influence the mean fundamental frequency of either the low- or high-frequency components of calls (Fig. 2). Testing using the mixed model concluded that for both the LFC and HFC, there was no consistent influence of caller sex on the fundamental frequency comparisons (LFC: $t_{310}=-1.212, p=0.23$; HFC: $t_{310}$ $=-1.207, p=0.23)$. 
TABLE I. Total number of calls by type recorded from each focal animal: $\mathrm{AF}$ - adult female, AM-adult male, and J-juvenile of either sex.

\begin{tabular}{lccccccccc}
\hline \hline & Age/Sex & N1 & N2 & N4 & N5 & N9 & N32 & N33 & Total \\
\hline A12 & AF & 1 & 6 & 1 & 1 & $\ldots$ & $\ldots$ & $\ldots$ & 9 \\
A23 & AF & $\ldots$ & 1 & $\ldots$ & $\ldots$ & 3 & $\ldots$ & $\ldots$ & 4 \\
A34 & AF & $\ldots$ & 2 & 3 & 5 & 1 & $\ldots$ & $\ldots$ & 11 \\
A8 & AF & $\ldots$ & 27 & 26 & 2 & 22 & $\ldots$ & $\ldots$ & 77 \\
W3 & AF & $\ldots$ & $\ldots$ & $\ldots$ & $\ldots$ & $\ldots$ & 2 & 5 & 7 \\
A27 & AM & $\ldots$ & 1 & $\ldots$ & $\ldots$ & $\ldots$ & $\ldots$ & $\ldots$ & 1 \\
A32 & AM & 4 & 7 & 10 & 8 & 19 & $\ldots$ & $\ldots$ & 48 \\
A33 & AM & $\ldots$ & $\ldots$ & $\ldots$ & 3 & $\ldots$ & $\ldots$ & $\ldots$ & 3 \\
A37 & AM & 9 & $\ldots$ & 15 & 13 & 7 & $\ldots$ & $\ldots$ & 44 \\
A38 & AM & $\ldots$ & 2 & 25 & $\ldots$ & 17 & $\ldots$ & $\ldots$ & 44 \\
A46 & AM & 2 & 6 & 11 & 9 & 8 & $\ldots$ & $\ldots$ & 36 \\
A6 & AM & 1 & $\ldots$ & 6 & $\ldots$ & 5 & $\ldots$ & $\ldots$ & 12 \\
W2 & AM & $\ldots$ & $\ldots$ & $\ldots$ & $\ldots$ & $\ldots$ & 3 & 17 & 20 \\
W5 & AM & $\ldots$ & $\ldots$ & $\ldots$ & $\ldots$ & $\ldots$ & $\ldots$ & 2 & 2 \\
A55 & J & 1 & 3 & 3 & 2 & 5 & $\ldots$ & $\ldots$ & 14 \\
A62 & J & $\ldots$ & $\ldots$ & 3 & $\ldots$ & $\ldots$ & $\ldots$ & $\ldots$ & 3 \\
Total & & 18 & 55 & 103 & 43 & 87 & 5 & 24 & 335 \\
\hline \hline
\end{tabular}

Orientation of the signaler, estimated from surfacing orientation, was a significant predictor of the HFC/LFC intensity ratio $\left(t_{184}=-2.64, p=0.009\right)$, while range and adult sex were not (Table II, column 1). There was a nonsignificant trend for HFC/LFC ratio to differ by adult sex $\left(t_{184}=-1.70\right.$, $p=0.091$; Fig. 3). The HFC/LFC ratio was higher when the caller was oriented more directly toward the hydrophone array (Fig. 3). Orientation was also a significant predictor $\left(t_{184}=-3.93, p=0.0001\right)$ of the number of modulations of the HFC fundamental by the LFC, with more sidebands observed when whales were oriented toward the hydrophone array, but range and adult sex were not (Table II, column 2).

Adult sex was a significant predictor of the relative level of the first two harmonics of the LFC $\left(t_{308}=-2.72, p\right.$ $=0.007$ ), while signaler orientation and range were not (Table II, column 3). Males had consistently more energy in the second harmonic than the fundamental across all seven call types, with an overall mean difference of $3.4 \mathrm{~dB}$ (Fig. 4).

\section{DISCUSSION}

Killer whale stereotyped calls appear to be an example of complex signals (Hebets and Papaj, 2005) with different information being encoded, and potentially transmitted,

TABLE II. Mean $\pm 95 \%$ confidence interval of spectral features of received calls by orientation, range, and adult sex. The units of the values in the table are the column unit divided by the row unit [e.g., for $-2.5 \pm 1.8 \mathrm{~dB} / \mathrm{rad}$, the difference in the HFC to LFC intensity ratio of calls produced by animals oriented directly toward the array $(0 \mathrm{rad})$ versus directly away $(\pi \mathrm{rad})$ is $-7.9 \pm 5.7 \mathrm{~dB}]$.

\begin{tabular}{lccc}
\hline \hline & HFC/LFC (dB) & HFC sidebands (n) & LFC h2/h1 (dB) \\
\hline Orientation (rad) & $-2.5 \pm 1.8^{\mathrm{a}}$ & $-0.43 \pm 0.21^{\mathrm{a}}$ & $-0.2 \pm 1.0$ \\
Range $(\log 10 \mathrm{~m})$ & $0.5 \pm 1.1$ & $-0.07 \pm 0.14$ & $0.1 \pm 0.6$ \\
Adult male relative & $-3.2 \pm 3.7$ & $-0.04 \pm 0.42$ & $2.8 \pm 2.0^{\mathrm{a}}$ \\
to adult female & & & 308 \\
Degrees of freedom & 184 & 184 & 308 \\
\hline \hline
\end{tabular}

${ }^{\mathrm{a}}$ Significant at $P<0.01$.

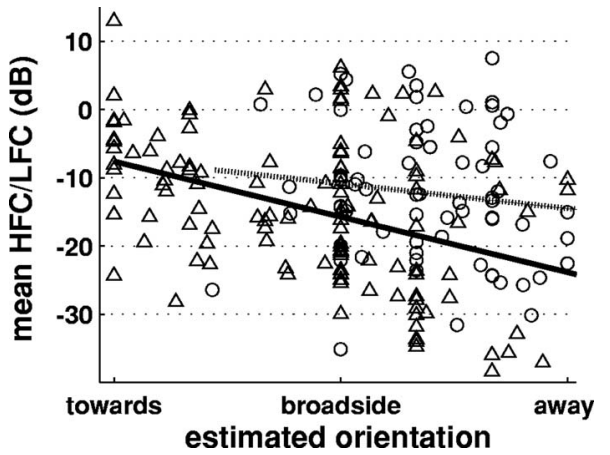

FIG. 3. The mean ratio of the peak energy of HFC to the summed energy in the first two harmonics of the LFC (HFC/LFC) versus estimated signaler orientation to the receiver based on surfacings before and after each call was produced. Calls of adult males are shown as triangles, with a solid regression line, while those of female are circles with a dashed regression line. Note the tendency for calls produced by adult males to have higher $\mathrm{HFC}$ / LFC ratios than calls produced by adult females.

within different call components. The intensity ratio of the first two LFC harmonics is significantly influenced by signaler sex, possibly because of the substantial body size differences in adult killer whales. On the other hand, the intensity ratio of the HFC relative to the $\mathrm{LFC}$ (HFC/LFC), and the number of HFC modulation bands, reflects the orientation of the signaler.

Interestingly, the fundamental frequency or pitch of both the LFC and HFC did not consistently vary between adult male and adult female body sizes (Fig. 2). Though diversity in mean fundamental frequency occurred within some call types in our sample (Fig. 2), there was no consistent pattern for either HFC or LFC across call types. Absolute frequency of the two components does not appear to be strongly influenced by sex and is probably constrained as stereotyped calls are shared within stable matrilineal groups.

Signaler orientation influenced the intensity of the HFC relative to the LFC, and the number of HFC sidebands, but not the relative intensity of the first two harmonics of the LFC (Table II and Fig. 3). These results indicate that lowfrequency adult sex cues may be more omnidirectional than other signal features. Miller (2002) found strong differences in the spectral structure of calls recorded from groups of whales moving toward versus away from the receiver. By

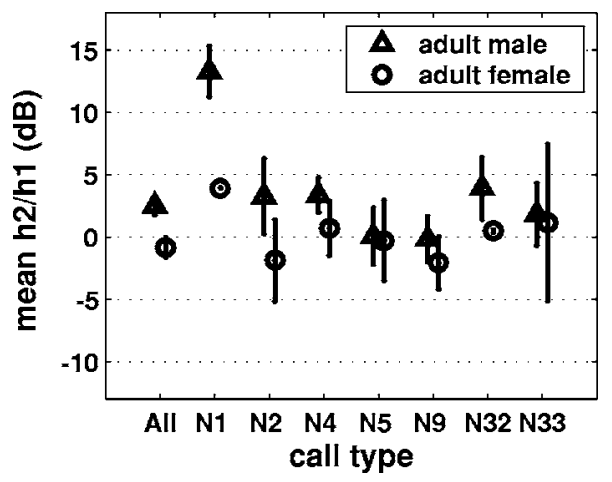

FIG. 4. The mean intensity ratio of the first two harmonics of the LFC (LFC $\mathrm{h} 2 / \mathrm{h} 1$ ) by call type for calls produced by adult males and adult females. The vertical bars are \pm 2 s.e. Note that the intensity ratio is greater in calls produced by adult males for all seven call types. 
measuring the energy in the fundamental of the HFC directly, this study confirms that this call component specifically encodes information on signaler orientation. In our sample, signaler orientation was estimated based on surfacing orientation before and after each call, so it would be interesting to investigate finer scale effects of orientation on HFC energy when signaler orientation at the time of the call is known with more precision. Higher frequencies of the HFC contour and the number of HFC sidebands would be predicted to be more directional than the LFC as there is more effective absorption, reflection, and focus of smaller wavelengths by sound source resonators and tissue structures. The ranges from caller to the hydrophone $(16-834 \mathrm{~m})$ seem to have little effect on the relative levels of the spectral features measured in this study, although range should strongly influence the absolute received levels of both the HFC and LFC as well as call reverberation patterns (Naguib and Wiley, 2001; Miller, 2002).

Adult sex influenced the relative energy in the first two harmonics of the LFC, but did not strongly influence the number of HFC sidebands (Table II). Our results may indicate that adult sex influences the HFC/LFC intensity ratio ( $p=0.091)$, but more data would be necessary to confirm this trend. Despite the mixed pattern in fundamental frequency, adult males had relatively more average energy in the LFC second harmonic than females across all seven analyzed call types (Fig. 4). Across all the 318 calls used in this study, the difference by adult sex in the relative levels of the first two harmonics averaged $3.4 \mathrm{~dB}$. As we also found that fundamental frequency within calls affects the relative level of the harmonics (see Sec. II), variation in the frequency content of analyzed calls probably dilutes the difference attributable to caller sex when values are averaged over the entire call, as was done in this study. Nonetheless, perception tests indicate that changes smaller than $3 \mathrm{~dB}$ in the relative levels of harmonics are detectable in birds and humans (Versfeld and Houtsma, 1995; Lohr and Dooling, 1998), and recent research suggests that a false killer whale (Pseudorca crassidens) can perceive small differences in relative harmonic intensities (Yuen et al., 2007). We can predict that the sex differences reported here should at least be detectable by the killer whales themselves. Playback experiments could test the salience of harmonic intensity patterns, and any role they might have in sex recognition.

\section{A. Implications for killer whale call production mechanism}

The presence of HFC sidebands at precise multiples of the LFC (Fig. 1) is evident that the physical process which generates the LFC also modulates the intensity of the HFC in some fashion. This interaction between the LFC and HFC seems to indicate that the two independent sources are in close proximity to each other within the whale. This is consistent with the possibility that the two contours are produced by independent, simultaneous control of the left and right monkey-lip dorsal bursae complexes, which are implicated in the production of sonar clicks of odontocetes (Cranford,
2000). However, alternative explanations for how these two independent contours might be produced cannot be ruled out.

We tentatively suggest that the sex differences in the relative intensity of the first two LFC harmonics may be explained by the presence of one or more resonant structures, which alter the relative intensities of the harmonics, and that males have lower-frequency resonances than females because of their larger size. We were able to measure the intensity of the first five LFC harmonics in 243 of the 318 calls produced by adult males or adult females (169 from adult males, 74 from adult females). Inspection of the relative energy in the first five harmonics indicated that frequencies within the second harmonic are supported in calls produced by males, while frequencies within the fourth harmonic are relatively more supported in calls from females. This seems to match predictions based on anatomy as adult males have roughly twice the body volume of adult females (Matkin and Leatherwood, 1986). In odontocetes several structures have been indicated as potential resonators, such as the nasal sacs or the dorsal bursae (Cranford, 2000; Cranford et al., 1996). If the size of these structures correlates with body size, then it could explain differences in resonances between adult male and female killer whales. However, more detailed knowledge of the sound production mechanism in killer whales would be necessary to identify the structures that do act as resonators and therefore to understand the influence of body size on the size of these structures.

Our interpretation that resonant effects influence the relative level of the LFC harmonics in killer whale calls is further supported by our finding that the relative intensity of the first two LFC harmonics changes with fundamental frequency within calls $(6.5 \mathrm{~dB} / \mathrm{kHz}, P<0.001$; see Sec. II $)$. The relative intensity of the harmonics changes as the frequencies excited by the harmonics change, which is consistent with the expected behavior of frequency-dependent resonant filters. Interestingly, time from the start of a call did not affect the relative intensity of the harmonics, suggesting that the movement of air during sound production itself does not strongly alter resonant characteristics in the soundproduction apparatus (Dormer, 1979). The observed differences in the relative levels of harmonics is consistent with the presence of resonances, but other analysis methodologies such as linear predictive coding (LPC; Fitch, 1997) are better suited to detect and quantify resonances. LPC is made difficult, though, in the case of killer whale calls because the fundamental frequency of the calls is quite high.

\section{CONCLUSIONS}

The sounds analyzed in this study were recorded from identified individuals during natural diving behavior, though the horizontal beamforming system does not allow measurement of caller depth directly. It is possible that, at fixed calling depths, sex or orientation differences might be greater than those observed here, and that natural variation in caller depth degrades these effects on the relative intensity of the harmonics. It would be useful to assess whether diving influences the features measured in this study using a method that can track the depth of calling animals. Such data could assess whether calling occurs in preferred depth bands (Hastie 
et al., 2006), which could be a simple way by which divers could reduce or enhance effects of diving on air-resonant properties, if they exist. Alternatively, it is possible that lowfrequency resonances could be under active control or occur in tissues rather than air, which should result in less sensitivity to caller depth. A heliox experiment with harbor porpoises indicated that the low-frequency component of clicks was based on air resonance and influenced by breathing heliox, but that high-frequency components were based in tissues and so uninfluenced (Amundin, 1991). Research on the mechanism by which the complex "two-voice" calls are produced could help elucidate the influence of diving ecology on acoustic signal structure, and the role of different signal features in communication.

\section{ACKNOWLEDGMENTS}

Thanks to the field and vessel crews during the fieldwork, to D. Bain for suggesting this analysis, and to V. Janik and P. Slater for useful comments. Monique MacKenzie of CREEM provided advice on the statistical analysis. A. Nousek, C. Wang, A. Hodgins-Davis, and S. Seneff at MIT's Laboratory for Computer Science helped build the corpus of sounds analyzed here. Funding was provided by WHOI's Rinehart Coastal Research Center and Ocean Ventures Fund, and a Royal Society USA/Canada fellowship to PJOM. All field recordings were conducted under a Canadian permit and followed Canadian law.

Amundin, M. (1991). "Helium effects on the click frequency spectrum of the harbor porpoise, Phocoena phocoena," J. Acoust. Soc. Am. 90, 53-59. Bradbury, J. W., and Vehrencamp, S. L. (1998). Principles of Animal Communication (Sinauer, Massachusetts)

Cranford, T. W. (2000). "In search of impulse sound sources in Odontocetes," in Hearing by Whales and Dolphins, edited by W. W. L. Au, A. N. Popper, and R. R. Fay (Springer, New York), pp. 109-155.

Cranford, T. W., Amundin, M., and Norris, K. S. (1996). "Functional morphology and homology in the odontocete nasal complex: Implications for sound generation," J. Morphol. 228, 223-285.

Deecke, V. B., Ford, J. K. B., and Spong, P. (2000). "Dialect change in resident killer whales: Implications for vocal learning and cultural transmission," Anim. Behav. 60, 629-638.

Dormer, K. J. (1979). "Mechanisms of sound production and air recycling in delphinids: Cineradiographic evidence,” J. Acoust. Soc. Am. 65, 229-239.

Fitch, W. T. (1997). "Vocal tract length and formant frequency dispersion correlate with body size in rhesus macaques," J. Acoust. Soc. Am. 102, 1213-1222.

Ford, J. K. B. (1987). "A catalogue of underwater calls produced by killer whales (Orcinus orca) in British Columbia," Can. Data Rep. Fish. Aq. Sci. No. 633.

Ford, J. K. B. (1991). "Vocal traditions among resident killer whales (Orcinus orca) in coastal waters of British Columbia," Can. J. Zool. 69, 14541483.

Ford, J. K. B., Ellis, G. M., and Balcomb, K. C. (2000). Killer Whales, 2nd ed. (Univ. of British Columbia, Vancouver).

Hastie, G. D., Wilson, B., and Thompson, P. M. (2006). "Diving deep in a foraging hotspot: Acoustic insights into bottlenose dolphin dive depths and feeding behaviour," Mar. Biol. (Berlin) 148, 1181-1188.

Hebets, E. A., and Papaj, D. R. (2005). "Complex signal function: Developing a framework of testable hypotheses," Behav. Ecol. Sociobiol. 57, 197-214.

Hoelzel, A. R., and Osborne, R. W. (1986). "Killer whale call characteristics: Implications for cooperative foraging strategies," in Behavioral Biology of Killer Whales, edited by B. C. Kirkevold and J. S. Lockard (Liss, New York, New York), pp. 373-403.

Janik, V. M., Sayigh, L. S., and Wells, R. S. (2006). "Signature whistle shape conveys identity information to bottlenose dolphins, "Proc. Natl. Acad. Sci. U.S.A. 103, 8293-8297.
Janik, V. M., and Slater, P. J. B. (2000). "The different roles of social learning in vocal communication," Anim. Behav. 60, 1-11.

Lammers, M. O., and Au, W. W. L. (2003). "Directionality in the whistles of Hawaiian spinner dolphins (Stenella longirostris): A signal feature to cue direction of movement?" Marine Mammal Sci. 19, 249-264.

Lohr, B., and Dooling, R. J. (1998). "Detection of changes in timbre and harmonicity in complex sounds by zebra finches (Taeniopygia guttata) and budgerigars (Melospittacus undulatus)," J. Comp. Physiol. 112, 36-47.

Masters, W. M., Raver, K. A. S., and Kazial, K. A. (1995). "Sonar signals of big brown bats, Eptesicus fuscus, contain information about individual identity, age and family affiliation," Anim. Behav. 50, 1243-1260.

Matkin, C., and Leatherwood, S. (1986). "General biology of the killer whale, Orcinus orca: A synopsis of knowledge," in Behavioral Biology of Killer Whales, edited by B. C. Kirkevold and J. S. Lockard (Liss, New York, New York), pp. 35-68.

McClelland, B. E., Wilczynski, W., and Ryan, M. J. (1996). "Correlations between call characteristics and morphology in male cricket frogs (Acris crepitans)," J. Exp. Biol. 199, 1907-1919.

Miller, P. J. O. (2002). "Mixed-directionality of killer whale stereotyped calls: A direction of movement cue?" Behav. Ecol. Sociobiol. 52, 262270.

Miller, P. J. O., and Tyack, P. L. (1998). "A small towed beamforming array to identify vocalizing resident killer whales (Orcinus orca) concurrent with focal behavioural observations," Deep-Sea Res., Part II 45, 13891405 .

Miller, P. J. O., and Bain, D. E. (2000). "Within-pod variation in the sound production of a pod of killer whales, Orcinus orca," Anim. Behav. 60, 617-628.

Miller, P. J. O., Shapiro, A. D., Tyack, P. L., and Solow, A. R. (2004). "Call-type matching in vocal exchanges of free-ranging killer whales, Orcinus orca," Anim. Behav. 67, 1099-1107.

Moors, H. B., and Terhune, J. M. (2005). "Calling depth and time and frequency attributes of harp (Pagophilus groenlandicus) and Weddell (Leptonychotes weddellii) seal underwater vocalizations," Can. J. Zool. 83, 1438-1452.

Naguib, M., and Wiley, H. (2001). "Estimating the distance to a source of sound: Mechanisms and adaptations for long-range communication," Anim. Behav. 62, 825-837.

Nousek, A. E., Slater, P. J. B., Wang, C., and Miller, P. J. O. (2006). "The influence of social structure on vocal signatures in northern resident killer whales (Orcinus orca)," Biol. Lett. 2, 481-484.

Olesiuk, P. F., Bigg, M. A., and Ellis, G. M. (1990). "Life history and population dynamics of resident killer whales (Orcinus orca) in the coastal waters of British Columbia and Washington state," Rep. Int. Whaling Com., Spec. Issue 12, pp. 209-243.

Pinheiro, J. C., and Bates, D. M. (2000). Mixed-Effects Models in S and S-Plus (Springer, New York).

$\mathrm{R}$ Development Core Team (2004). R: A language and environment for statistical computing, (version 1.9.1), R Foundation for Statistical Computing, Vienna, Austria, ISBN 3-900051-00-3, URL http://www.Rproject.org, viewed 4/20/2007.

Rendall, D., Kollias, S., Ney, C., and Lloyd, P. (2005). "Pitch $\left(F_{0}\right)$ and formant profiles of human vowels and vowel-like baboon grunts: The role of vocalizer body size and voice acoustic allometry," J. Acoust. Soc. Am. 117, 944-955.

Ridgway, S. H., Carder, D. A., Kamolnick, T., Smith, R. R., Schlundt, C. E., and Elsberry, W. R. (2001). "Hearing and whistling in the deep sea: Depth influences whistle spectra but does not attenuate hearing by white whales (Delphinapterus leucas) (Odontoceti, Cetacea)," J. Exp. Biol. 204, 38293841.

Tyack, P. L. (1991). "If you need me, whistle," Nat. Hist. Aug, 60-61.

Tyack, P. L., and Miller, E. H. (2002). "Vocal anatomy, acoustic communication and echolocation," in Marine Mammal Biology: An Evolutionary Approach, edited by A. R. Hoelzel (Blackwell Science, Oxford, UK), pp. $142-184$.

Versfeld, N. J., and Houtsma, J. M. (1995). "Discrimination of changes in the spectral shape of two-tone complexes," J. Acoust. Soc. Am. 98, 807816.

Wang, C., and Seneff, S. (2000). "Robust pitch tracking for prosodic modeling in telephone speech," in IEEE International Conference on Acoustics, Speech and Signal Processing, Istanbul, Turkey.

Yuen, M. M. L., Nachtigall, P. E., Breese, M., and Vlachos, S. A. (2007). "The perception of complex tones by a false killer whale (Pseudorca crassidens)," J. Acoust. Soc. Am. 121, 1768-1774. 